\title{
Graphene and Its Derivates, Applications and Environmental Issues
}

\author{
Z. Sofer ${ }^{1}$, D. Sedmidubský ${ }^{1}$, J. Luxa ${ }^{1}$, O. Jankovský ${ }^{1}$, D. Bouša ${ }^{1}$, K. Klímová ${ }^{1}$, and M. Pumera ${ }^{2}$ \\ ${ }^{1}$ Dept. of Inorganic Chemistry, University of Chemistry and Technology Prague, Technická 5, 16628 Prague 6, Czech \\ Republic, zdenek.sofer@vscht.cz \\ ${ }^{2}$ School of Physical \& Mathematical Sciences, Nanyang technological University, 637371, Singapore
}

Graphene is one of the wonder materials of the current science. Due to its unique properties this material has a huge application potential covering not only electronics and catalysis, but also environmental and biomedical applications. The huge application potential of graphene is based, among others, on its large surface area and affinity towards many metallic ions. This makes graphene an ideal material for sorption applications. The sorption on graphene and in particular on graphene oxide surface is related to the presence of oxygen functionalities which can coordinate and successfully entrap various metallic ions. This represents a great advantage in comparison with other carbon materials, where usually only physisorption takes place. Another great advantage originates from the possibility of relatively simple surface modification leading to an increased sorption capacity, or to high selectivity towards various ions. This offers a great opportunity not only for environmental science, but it can be also exploited in other applications like selective trapping of rare metals occurring at low concentrations. On the other hand the synthesis of graphene on large scale can bring several disadvantages. The synthetic methods based on thermal reduction led to evolution of large amount of organic compounds, some of, which are highly toxic, like benzene and some of its derivates. The optimization of synthetic procedures can partially overcome this issue, however it must be considered for the large production. Due to the strong interaction of graphene skeleton with organic molecules, mainly with aromatic system of electrons, the organic exfoliation products remain adsorbed on graphene surface and high temperature treatment of graphene leads to their evolution. 